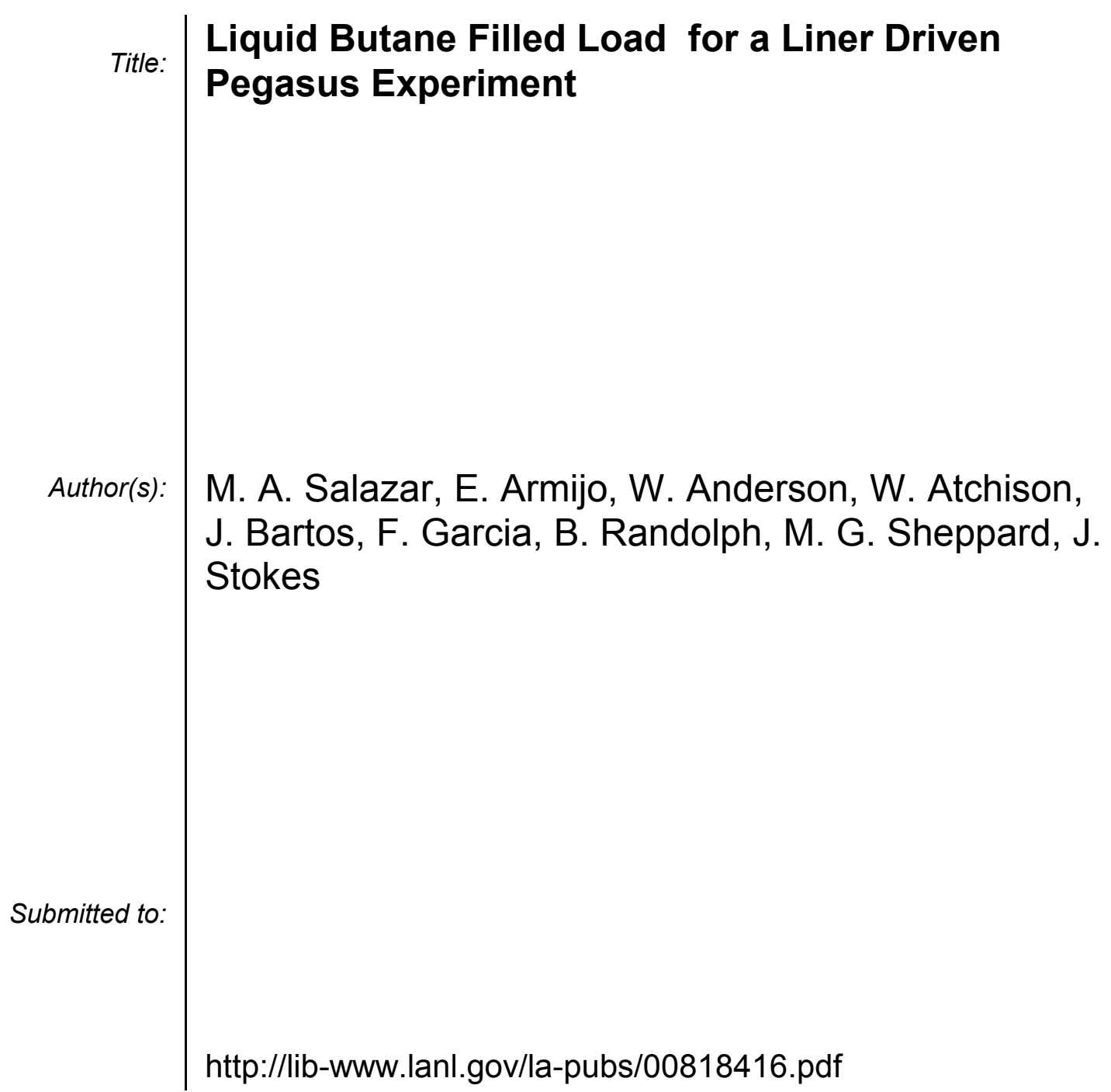




\title{
LIQUID BUTANE FILLED LOAD FOR A LINER DRIVEN PEGASUS EXPERIMENT
}

\author{
M. A. Salazar, E. Armijo, W. Anderson, W. Atchison, \\ J. Bartos, F. Garcia, B. Randolph, M. G. Sheppard, J. Stokes \\ Los Alamos National Laboratory, MS E-549, Los Alamos, New Mexico, 87545, U.S.A.
}

LA-UR- 01-3226

\section{Abstract}

A hydrogen rich, low density liquid, contained within the internal volume of a cylindrical liner, was requested of the Polymers and Coatings Group (MST-7) of the Los Alamos Materials Science Division for one of the last liner driven experiments conducted on the Los Alamos Pegasus facility. The experiment (Fig.1) was a continuation of the Raleigh-Taylor hydrodynamics series of experiments and associated liners that have been described previously $[1,2]$.

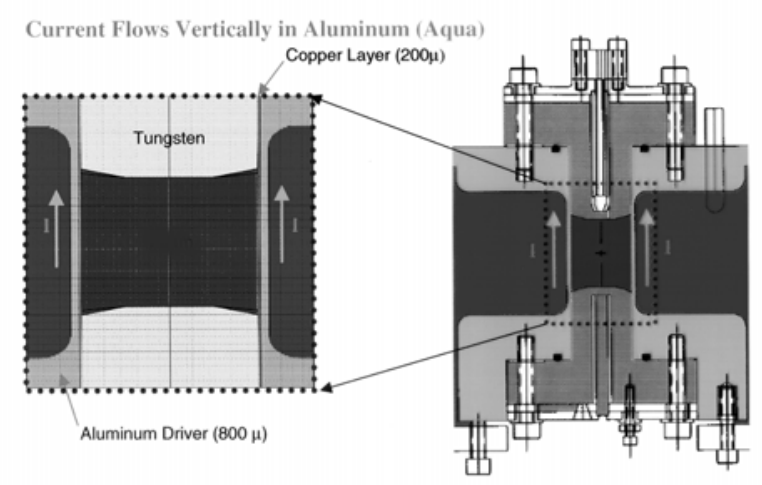

Figure 1. Experiment Diagram.

These experiments required massive tungsten glide planes for inertial confinement of the liner fill media during implosion. Shallow sinusoidal perturbations were machined on the inside surface of the liner to seed instabilities, also true of the previous experiments. Butane was selected for a relatively low equilibrium vapor pressure, a practical attribute for use in the Pegasus vacuum power flow channel. Butane safety topics at Pegasus are addressed. Glide planes were sealed to the liner by use of butane compatible o-rings. A sintered form of tungsten was used for the glide planes to facilitate machining the relatively complex shapes that were required. Porosity of the tungsten was sealed by an epoxy vacuum/pressure impregnation procedure. Following some development experiments, we chose to pre-fill the load with butane and seal the liner assembly as opposed to filling the load after being installed in the target chamber at Pegasus. A butane reservoir was developed and mounted above the load as a part of the load assembly to allow monitoring the liquid level and to prevent loss of the entire experiment as a result of possible butane loss during the few days required to set up a Pegasus experiment. This paper describes the load fabrication processes and development of novel procedures that were required. We expect to apply the technologies to future Los Alamos experiments that will be conducted at the new Atlas facility.

\section{INTRODUCTION}

Pegasus experiment RTMIX-5 was designed to investigate seeded instability growth at an interface between a soft metal alloy ( $\mathrm{Sn} / \mathrm{In})$ and a hydrogen rich volatile liquid (condensed butane, $\mathrm{C}_{4} \mathrm{H}_{10}$ ) [3]. Approximately $10 \mathrm{~cm}^{3}$ of liquid butane $(\sim 5 \mathrm{~g}$ at $\sim 0.5$ $\mathrm{g} / \mathrm{cm}^{3}$ ) was vaporized inside the Pegasus vacuum chamber in conducting this experiment. This release was expected to occur about $50 \mu \mathrm{s}$ after current start. A primary goal in designing the experiment was to make sure that personnel, equipment and data were protected in the unlikely event of butane combustion.

\section{TEST FILL APPARATUS}

The test fill apparatus is shown in Fig.2. An acrylic chamber duplicates the dimensions of the experimental volume. It uses the tungsten glide planes of the experiment and an acrylic reservoir for containing a visible head of liquid butane above the liner experiment. This device revealed the tungsten glide planes leaked through the porosity of the sintered metal. Oil seeped from the pores after exposure to liquid butane. Pore sealing techniques were developed as described below. Techniques for butane filling and for keeping bubbles out of the test volume were developed with this apparatus in a fume hood. Vacuum was applied to the test volume to mimic the Pegasus vacuum power flow channel. Butane was introduced at the bottom of the liner volume. Venting at the top valve of the reservoir was required to fill the two volumes. The target volume was cooled with sprayed canned air to condense butane without trapping bubbles. To prevent bubble formation within the test volume after 
filling, 35 psi of Argon pressure was applied at the top of the reservoir.

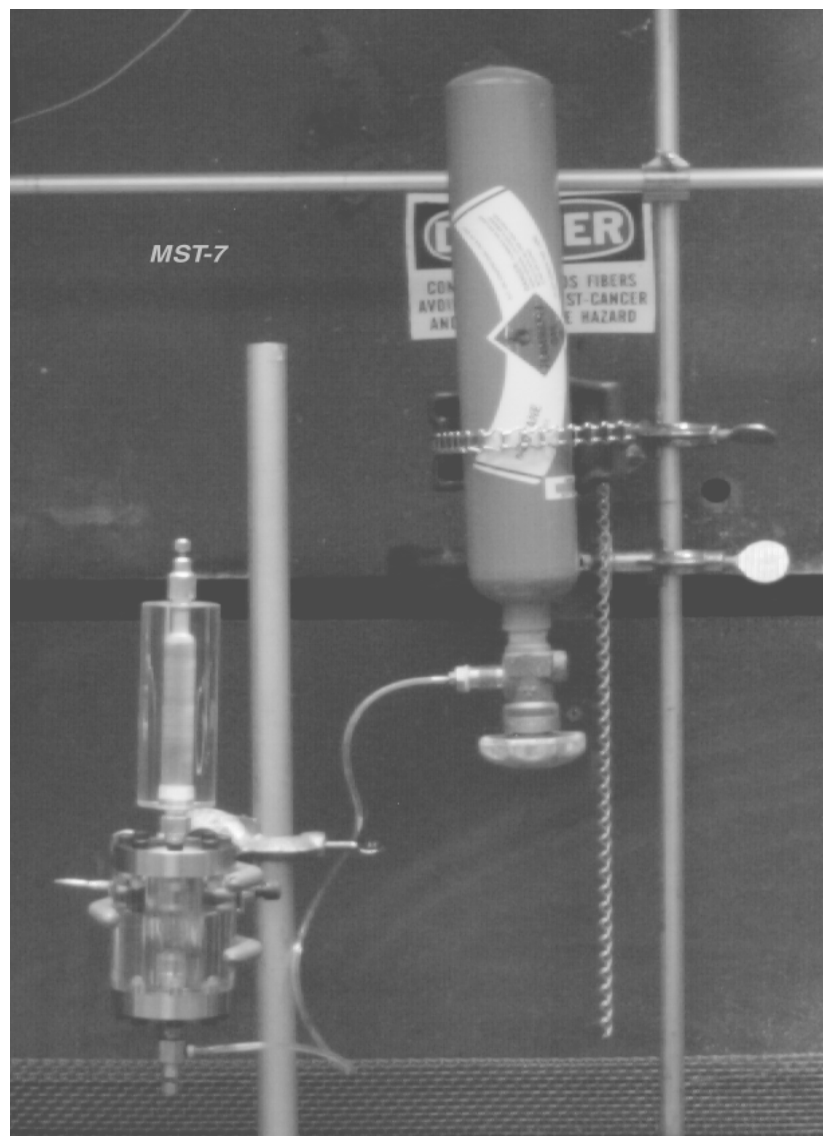

Figure 2. Test fill apparatus.

\section{EPOXY IMPREGNATION PROCEDURE}

Two-component epoxy resin (Epon 815 and TETA) at $14 \mathrm{phr}$ was used to fill the porosity of the glide planes. This was done by fully immersing the glide planes in a pot of the mixed resin. The apparatus containing the pot was first evacuated to remove gas from the pores and then over pressured with air at 100 psi to drive epoxy resin into the pores of the tungsten.

The epoxy resin used was exothermic, has a very short pot life, and sets up much more quickly than we would have desired. We have subsequently obtained a curing agent that is far less reactive to provide better control over any similar impregnation procedures in the future. After curing, the glide planes were removed from the epoxy block by smacking the block with a hammer to fracture the brittle epoxy. This worked well to remove the excess epoxy.

Upon assembling the experiment we found that we had not entirely plugged the porosity. Some butane leakage still occurred through the sintered tungsten glide planes.
Using the same epoxy material and similar techniques as before, the volumes around the tungsten at the ends of the assembled liner were filled with epoxy and the leakage was stopped. The epoxy can be seen at the upper end of the liner in Fig. 3.

The epoxy becomes hot while curing. This observation created concern that the low melting point indium tin alloy sleeve within the aluminum liner might melt and flow during potting. The liner was x-radiographed after potting and no change in the indium tin sleeve was detected.

\section{IV.BUTANE FILLED EXPERIMENT}

The actual target and reservoir (Fig. 3) was filled in a laboratory at the Target Fabrication Facility (TFF). The target assembly was moved while filled with butane to the Pegasus machine and installed for the experiment in a building near the TFF. Compressed argon was introduced through a nipple at the top of the reservoir after the target assembly was installed. The level of butane in the reservoir dropped back to the original fill level upon applying argon pressure to the reservoir. This is interpreted as confirmation that the test volume was filled with liquid and all vapor bubbles were condensed back to liquid.

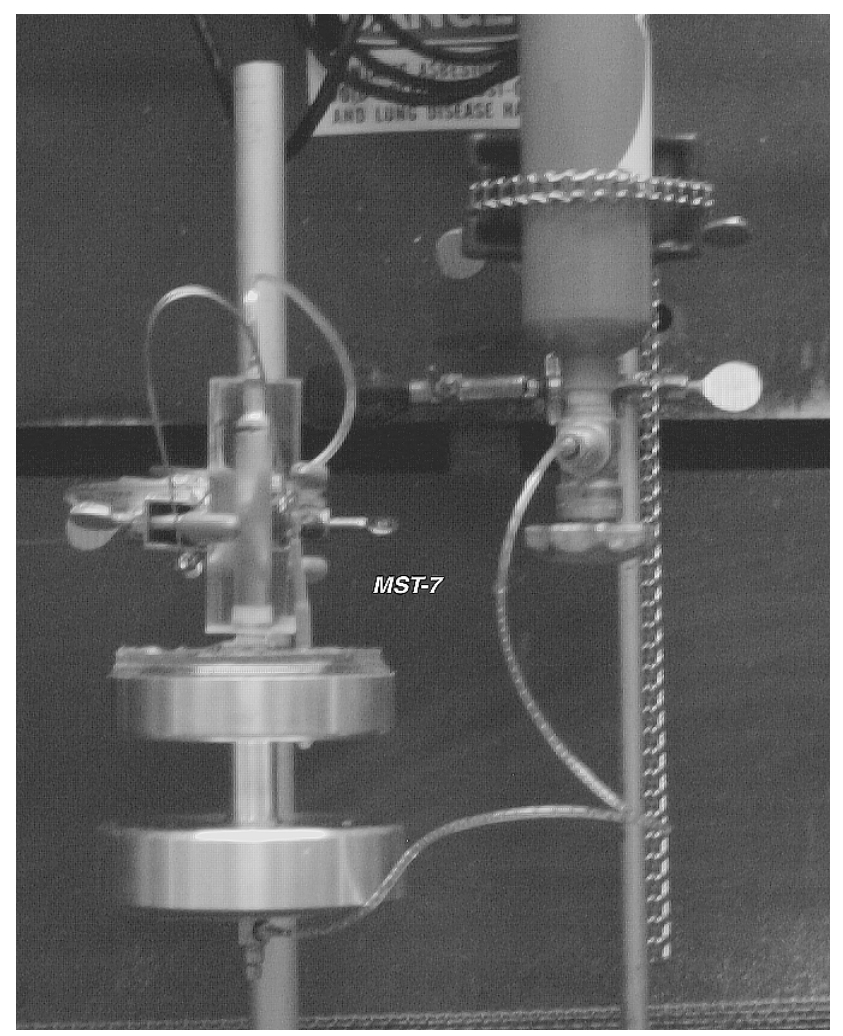

Figure 3. RTMIX5 load with reservoir. 


\section{BUTANE SAFETY}

At the conclusion of the RTMIX-5 experiment about 5 $\mathrm{g}$ of heated butane vapor was released to the Pegasus vacuum chamber. Prior to conducting the experiment butane safety issues were evaluated. Since the partial pressure of the heated, flammable gas would be much lower than the ambient air pressure, a target chamber breach from shot damage would transport air from an infinite supply into the vicinity of the leak within the chamber. The initial velocity of debris from z-pinch experiments is rarely in any direction other than along the cylindrical liner axis, vertical in the case of Pegasus. If a vacuum leak were to occur, it would be expected to occur at the top or bottom of the vacuum chamber. The potential for developing an explosive butane/air mixture then is most probably at the vacuum chamber ends.

The primary butane safety plan was to first prevent a vacuum breach, and then to flood the chamber with sufficient $\mathrm{CO}_{2}$ immediately after the experiment so that any subsequent mixing with air would always result in a butane $/ \mathrm{CO}_{2} /$ air mixture that is too lean to burn. Protection against vacuum breaks was accomplished by putting debris catchers inside the vacuum chamber at the top and bottom

\section{A. Safe Pegasus Vacuum Chamber Venting}

A remotely operated valve was opened shortly after the experiment to flood the chamber with $\mathrm{CO}_{2}$. An input $\mathrm{CO}_{2}$ flow-rate of 300 liters/minute will give about 5 vacuum chamber gas exchanges in 15 minutes. A directional valve on the chamber allowed the $\mathrm{CO}_{2}$ /butane mixture to be vented out of the building when the chamber pressure exceeded one Atm pressure. The one-way valve, which supports vacuum in the chamber was set to open with only a slight over-pressure in the chamber

As an extra safety precaution, the exhaust line gas was further diluted with $\mathrm{CO}_{2}$ before reaching the end of the vent line where the exhausted gas could mix with air. The exhaust-gas-line diluent was turned on a few minutes before the experiment. The exhaust line was calculated to be able to handle flow-rates of twice the chamber input $\mathrm{CO}_{2}$ flow rate without a large pressure differential. The exhaust line diluent flow-rate was set to about $10 \%$ of the chamber input $\mathrm{CO}_{2}$ flow-rate.

\section{B. Venting After Shot Damage, Small Leak}

In the event the debris catchers fail and vacuum is broken during the experiment, it will most likely happen at the top or bottom of the chamber for the reasons previously stated. The top and bottom of the vacuum chamber were each covered with $\mathrm{CO}_{2}$ filled balloons containing enough total volume of $\mathrm{CO}_{2}$ to dilute butane in the vacuum chamber to a safe mixture before dangerous levels of $\mathrm{O}_{2}$ from air could enter the chamber. For small leaks, chamber venting would still proceed primarily as described above. Any gas mixture that diffused back through the vacuum leak into the Pegasus building after the vessel reached ambient pressure would be much too lean to burn and would be removed from the building with existing exhaust fans. Since the mixture would not be flammable, standard ventilation systems are adequate.

In the event that a vacuum breach occurred at a place other than the top or bottom of the chamber, where no $\mathrm{CO}_{2}$ filled balloons were present, air would enter the chamber. For a small leak, the $\mathrm{CO}_{2}$ flood would still dilute the mixture enough to ensure that flammable conditions were avoided, and flushing would proceed as described above.

\section{Venting After Shot Damage, Large Leak}

In the event of a catastrophic failure at a point not protected by $\mathrm{CO}_{2}$ balloons, the chamber would rapidly fill with room air. If the chamber were to reach ambient pressure with the air and flammable gas isotropically mixed, assuming no dilution with $\mathrm{CO}_{2}$, the resulting mixture would still be too lean to burn. However, during filling, an improbable transient flammable condition could occur. If ignition also occurred, pressure from combustion would drive gases back into the room through the catastrophic failure point in the vacuum chamber. Once in the room, the mixture would immediately become too lean to support combustion. Inside the vessel, we expect the combustion would quickly exhaust the fuel or the $\mathrm{O}_{2}$ supply from air, much like the "poof" that happens when lighting a propane grill after letting the gas build up a little too long. The maximum energy release in this case is calculated to be $250 \mathrm{~kJ}$. The calculation assumes $100 \%$ burn efficiency which requires all of the butane to be simultaneously mixed with air in locally flammable concentrations at the time of ignition; not a likely scenario! Since there are likely to be many hot sources for ignition inside the chamber after an experiment, our expectation is that as soon the local mixture at any one of the hot spots became flammable, ignition would occur. Then, only the small quantity of butane locally mixed with air in flammable concentrations at the time of ignition would burn.

The chance that ignition would be delayed until conditions for detonation could be established, and that ignition would also occur, both before uniform mixing and pressure equilibration with the room resulted in a "too-lean" non-flammable mixture, is considered to be vanishingly small. If a stoichiometric mixture of air and butane did volume detonate to completion inside the vessel, releasing $250 \mathrm{~kJ}$ of combustion energy, we estimate that the maximum over-pressure produced outside the chamber wall would be only $0.33 \mathrm{psi}$, considered a safe level.

\section{VI.SUMMARY AND CONCLUSIONS}

Cylindrical hydrodynamic experiment loads can be designed and fabricated to take advantage of the material properties of flammable liquids. Safety in handling flammable liquids and gasses is of paramount importance. The techniques to handle and fill such loads are 
manageable in a laboratory setting and during a liner zpinch experiment. The risk of hazardous combustion is very small, even in the occurrence of a vacuum chamber breach due to shrapnel damage during a Pegasus experiment.

\section{REFERENCES}

[1] Maurice Sheppard, Walter Atchison, Rhon Keinigs and John Stokes, "Rayleigh-Taylor Instability Growth Enigma: Liner Studies on Pegasus". in Proc. $12^{\text {th }}$ IEEE International Pulse Power Conference, ed. C. Stalling and H. Kirbie, 1999. p. 892.

[2] W. L. Atchison, W. Anderson, R. Keinigs, D. Oro, M. Salazar, M. Sheppard, and J. Stokes, "Summary of the Raleigh-Taylor Instability Studies at the Pegasus Facility," Paper O3B1, this conference.

[3] B. Randolph, "Fabrication Process for Machined and Shrink Fitted Impactor-type Liners for the Los Alamos HEDH Program," to be published in Proc. VIIIth International Conference on Megagauss Magnetic Field Generation and Related Topics, 1998. 